\title{
A parallel image encryption algorithm based on the piecewise linear chaotic map and hyper-chaotic map
}

\author{
Yuling Luo - Ronglong Zhou - Junxiu Liu - Yi Cao - Xuemei Ding
}

the date of receipt and acceptance should be inserted later

\begin{abstract}
This paper proposes a parallel digital image encryption algorithm based on a piecewise linear chaotic map (PWLCM) and a four-dimensional hyper-chaotic map (FDHCM). Firstly, two decimals are obtained based on the plain-image and external keys, using a novel parallel quantification method. They are used as the initial value and control parameter for the PWLCM. Then, an encryption matrix and four chaotic sequences are constructed using the PWLCM and FDHCM, which control the permutation and diffusion processes. The proposed algorithm is implemented and tested in parallel based on a Graphics Processing Unit (GPU) device. Numerical analysis and experimental results show that the proposed algorithm achieves a high encryption speed and a good security performance, which provides a potential solution for real-time image encryption applications.
\end{abstract}

Keywords image security; chaotic encryption; parallel encryption; GPU; security analysis

Yuling Luo, Ronglong Zhou, Junxiu Liu

Guangxi Key Lab of Multi-Source Information Mining and Security, Faculty of Electronic Engineering, Guangxi Normal University, Guilin, China

E-mail: liujunxiu@mailbox.gxnu.edu.cn

Yi Cao

Department of Business Transformation and Sustainable Enterprise, Surrey Business School, University of Surrey, Surrey, United Kingdom, GU2 7XH.

Xuemei Ding

School of Computing, Engineering and Intelligent Systems, Ulster University, United Kingdom, BT48 7JL; and Faculty of Software, Fujian Normal University, Fuzhou 350108, China.

\section{Introduction}

The rapid and continual transmissions of digital information on the internet have had a revolutionary impact. Providing digital information security in the transmission channel has become an increasingly serious issue because the data can be intercepted, cracked, or destroyed [14,39]. The number of image files transmitted on the Internet continues to increase. Due to bulky data capacity of images, high redundancy and strong correlations among adjacent pixels, some traditional encryption algorithms, e.g., the preliminary techniques Data Encryption Standard (DES), Advanced Encryption Standard (AES), International Data Encryption Algorithm (IDEA) and Rivest Shamir Adleman (RSA) algorithms, are not efficient for image encryption [42]. Chaotic systems have many excellent intrinsic properties, including ergodicity, non-periodicity, non-convergence and sensitiveness on initial conditions, which have attracted significant attention for the design of new image cryptosystems 20,27,28, 35.

Many chaos-based image encryption algorithms have been proposed in terms of the permutation and diffusion of Shannon encryption architecture. Ref 29] firstly proposed a logistic map-based encryption algorithm. Since then, a variety of novel chaotic systems and chaos-based image encryption methods have been designed to be applied for the secure communications, including the logistic map $13,24,26$, the Chua system [4], the Chen system [37, the Arnold cat map 12 and hyper-chaotic maps $17,21,41$. Specifically, several chaotic cryptosystems have been proposed for image security 23,30$]$. However there still exist some common drawbacks for image secure application, such as the frangibility of resisting 
known-plaintext attack and differential cryptanalysis [6, 39], short periodic, the incomplete effect for the decryption images, and excessive time for encryption or decryption process 22,30 which is not efficient for the secure transmission of image data.

In order to improve the mentioned-above shortages, Ref. 30 proposed a parallel encryption method for the image data, which is based on a total shuffling operation. Specifically, the plain-image is firstly divided into four sub-images, and the geometric space distribution of each sub-image is pseudo-randomly disturbed in terms of the logistic map. Meantime, a total shuffling matrix is used to shuffle the pixel position of the entire image, and the sub-images are encrypted simultaneously in parallel. However, due to its simple structure, the total shuffling process is not secure because the encryption algorithm cannot effectively resist the plain-text attack owing to the shuffling process of each sub-image before that of the total image. Besides, the execution time of the total process is still a bit high to some extent 2. And Ref. 11 proposed an efficient and secure encryption scheme for medical images based on chaotic maps, which consists of a bit-level substitution mechanism to simultaneously achieve the effect of confusion and diffusion to improve the processing speed. However, in Ref. 7, the authors proved that the scheme cannot resist differential cryptanalysis because the security of the original scheme [11 only depends on the permutation key instead of on all of the keys which make the key space greatly reduced, and the permutation key is not sensitive to the plain-text to further weaken the capability of resisting the differential attack. As we all known, differential cryptanalysis is usually a chosen plain-text attack which is the most threatening form of attack among the classical known plain-text attack, known cipher-text attack, and chosen plain-text attack, and the basic method of differential cryptanalysis for the image encryption algorithm is to use pairs of plain-text related by a constant difference to computer the differences of the corresponding cipher-texts and to detect the statistical distribution of the key. Therefore, the sensitivity degree to the plain-text for an image encrypted scheme is usually utilized to test the robustness of the encrypted scheme to resist the differential cryptanalysis, in that, it is used to judge whether the encrypted images are totally different or not even though there is only 1-bit change for original two plain-texts. Thus, it is very important to have a good sensitivity to the plain-image information for the cryptosystems. Moreover, in order to further improve the speed of the chaos-based cryptosystem, two new parallel image encryption algorithms have been proposed in the past few years 15, 18. Specifically, compressive sensing is applied for the encryption scheme in Ref. 15, and a reversible one-dimensional cellular automata is used to design the parallel scheme in Ref. [18]. The common feature of these two encryption schemes is to divide the image into several fixed-size blocks to encrypt in parallel. Comparing these two schemes, the encryption scheme in Ref. 18 has better security than that in Ref. [15 even though the encryption time of Ref. [15] is shorter than that of Ref. [18, and the reason is that a lossy compression method has been used for the scheme in Ref. 15 to reduce the computing time, which makes the quality of the decrypted image become worse due to the implementation of a lossy compression on the platform of the multi-threading CPU devices.

Comparing to $\mathrm{CPU}$, the GPU is more suitable to accelerate encryption for large dataset because it has more computing units 32,40$]$. So some parallel image encryption schemes are now considered to be implemented on the GPU platform 19,33 . Besides, a series of performance analysis results, including algorithm complexity, speedup, redundancy and code execution efficiency etc., have demonstrated that GPU performance is superior to the CPU. Ref. 19] used the GPU to accelerate a double random phase encoding (DRPE) algorithm for image's fast encryption, and the experimental results showed that the speedup between parallel and serial computing is amplified with an increase in image size, which means that the acceleration effect increases. And in Ref [33], the author proposed a high speed counter cryptography mode based on GPU parallelism. Specifically, it focuses on the symmetric-key cryptography that uses modern block cipher with a fixed size of data. The implementation results illustrate that the GPU is a suitable technology which can significantly improve the computational performance for the encryption and decryption of image data.

Based on the above analysis, a good parallel encryption system should not only ensure security and robustness to defend from cryptanalysis attacks, but also have a fast encryption speed to be beneficial for the real-time transmission of image in practical application. In this paper, a parallel image encryption mechanism based on the chaotic map is proposed, and this mechanism is evaluated by using a GPU device. The main contributions of this paper include the followings: (1) a novel parallel decimal quantification method based on a plain-image and an initial key; (2) a new encryption matrix based on a parallel generated chaotic sequence; (3) a novel customized parallel 
permutation-diffusion approach; (4) an image encryption approach implemented on the GPU based on OpenCL technology; and (5) a detailed security analysis of the proposed scheme.

The rest of this paper is organized as follows. Section 2 describes two chaotic maps of the PWLCM and FDHCM, the preliminary knowledge of the decimal quantification process, and the construction of the encryption matrix. Section 3 reveals the proposed cryptosystem in detail. Section 4 gives the implementation method based on a GPU device using the OpenCL technology. Section 5 provides the security performance analysis. The final section concludes the paper.

\section{Preliminaries}

In this section, we mainly introduce two chaotic maps of the PWLCM and FDHCM, a novel decimal parallel quantification process based on the plain-image and external key, and the construction of encryption matrix.

\subsection{Two chaotic maps}

The piecewise linear chaotic map (PWLCM) is given by

$$
\begin{aligned}
x(i+1) & =F(x(i), \mu) \\
& = \begin{cases}\frac{x(i)}{\mu} & \text { if } x \in(0, \mu] ; \\
\frac{x(i)-\mu}{2-\mu} & \text { if } x \in(\mu, 0.5] ; \\
F(1-x(i), \mu) & \text { if } x \in(0.5,1),\end{cases}
\end{aligned}
$$

where $x(i) \in(0,1)$. When the control parameter $\mu \in(0,0.5)$, it evolves into a chaotic state, and $\mu$ can serve as a secret key. The PWLCM system has a uniform invariant distribution and very good ergodicity, confusion and determinacy. Therefore it can provide an excellent random sequence that is suitable for the cryptosystem.

The four-dimensional hyper-chaotic map (FDHCM) used in this paper is given by

$$
\left\{\begin{array}{l}
\dot{x_{1}}=a\left(x_{2}-x_{1}\right)+x_{2} x_{3} x_{4} ; \\
\dot{x_{2}}=c x_{1}-x_{2}-x_{1} x_{3} x_{4} ; \\
\dot{x_{3}}=-b x_{3}+x_{1} x_{2} x_{4} ; \\
\dot{x_{4}}=d x_{1}+x_{1} x_{2} x_{4},
\end{array}\right.
$$

where $x_{1}, x_{2}, x_{3}, x_{4}$ are state variables and $a, b, c, d$ are real constant parameters. Compared with low-dimensional chaotic maps, high-dimensional chaotic maps (especially hyper-chaotic systems) have a larger key space, better sensitivity, more complex dynamic characteristics and randomness. The low-dimensional chaotic maps can be deciphered using phase space reconstruction and nonlinear prediction techniques etc., however those techniques cannot decipher the high-dimensional chaotic systems 25 . We choose $a=10, b=3 / 8, \quad c=28, d=-1$, the initial conditions are $(1,1,1,1)$, and the time step size is 0.0001 . Its chaotic behaviour portraits are shown in Fig. 1.
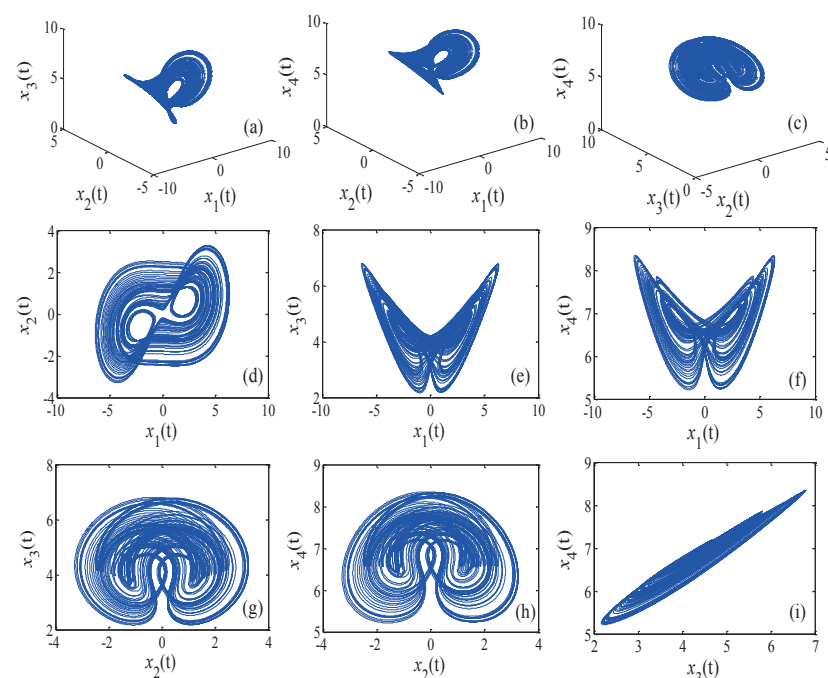

Fig. 1 Phase portraits of FDHCM with parameters $a=10$, $b=3 / 8, c=28, d=-1$. (a)-(c) are $3 \mathrm{D}$ views in the $x_{1}-x_{2}-x_{3}$, $x_{1}-x_{2}-x_{4}$ and $x_{2}-x_{3}-x_{4}$ spaces. (d)-(i) are projections on the $x_{1}-x_{2}, x_{1}-x_{3}, x_{1}-x_{4}, x_{2}-x_{3}, x_{2}-x_{4}, x_{3}-x_{4}$ planes.

\subsection{A novel parallel decimal quantification}

According to the external keys and plain-image information, a novel parallel quantification method is designed to obtain the decimal number in the range of $(0,1)$, which is used to be the initial value of the PWLCM to generate chaotic sequences. In this way, the generated sequence will be strictly related to the

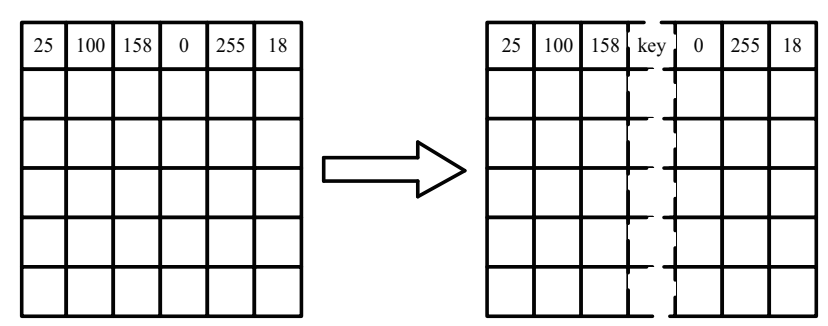

Fig. 2 A $6 \times 6$ matrix and an $s$ byte key. 


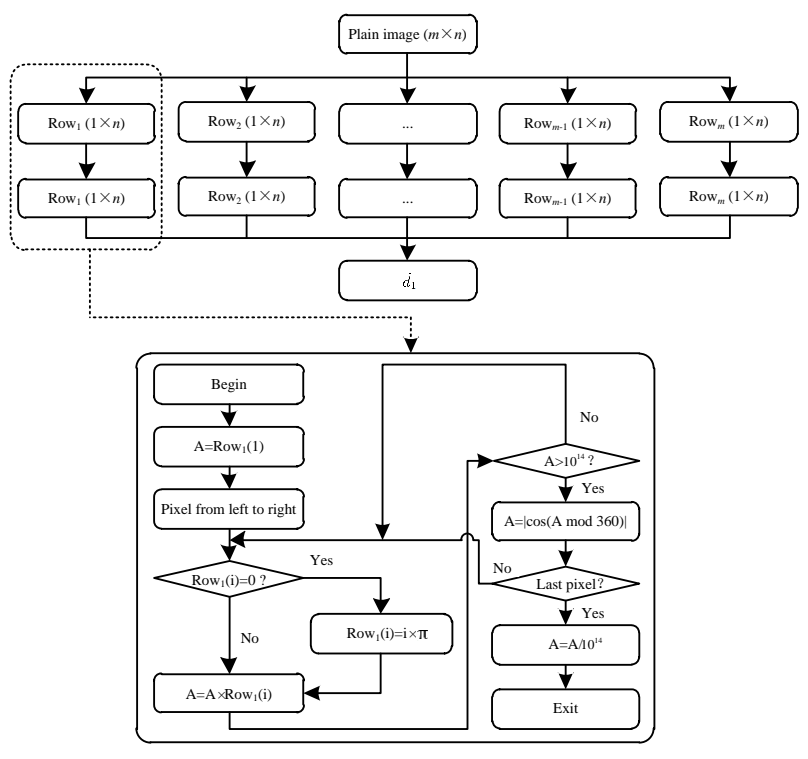

Fig. 3 The flow chart of the quantification process.

\begin{tabular}{|c|c|c|c|c|c|c|}
\hline$\left(\mathbf{x}_{1}(201), \mathbf{x}_{2}(201)\right)$ & & $\mathbf{Y}_{1}(1)$ & $\mathbf{Y}_{1}(2)$ & $\cdots$ & $\mathbf{Y}_{1}(n-1)$ & $\mathbf{Y}_{1}(n)$ \\
\hline$\left(\mathbf{x}_{1}(202), \mathbf{x}_{2}(202)\right)$ & 8 & $\mathbf{Y}_{2}(1)$ & $\mathbf{Y}_{2}(2)$ & $\cdots$ & $\mathbf{Y}_{2}(n-1)$ & $\mathbf{Y}_{2}(n)$ \\
\hline$\vdots$ & & $\vdots$ & $\vdots$ & $\vdots$ & $\vdots$ & $\vdots$ \\
\hline$\left(\mathbf{x}_{\mathbf{1}}(199+m), \mathbf{x}_{2}(199+m)\right)$ & $\rightarrow$ & $\mathbf{Y}_{m-1}(1)$ & $\mathbf{Y}_{m-1}(2)$ & $\cdots$ & $\mathbf{Y}_{m-1}(n-1)$ & $\mathbf{Y}_{m-1}(n)$ \\
\hline$\left(\mathbf{x}_{1}(200+m), \mathbf{x}_{2}(200+m)\right)$ & & $\mathbf{Y}_{m}(1)$ & $\mathbf{Y}_{m}(2)$ & $\cdots$ & $\mathbf{Y}_{m}(n-1)$ & $\mathbf{Y}_{m}(n)$ \\
\hline
\end{tabular}

Fig. 4 The encryption matrix generation process.

external keys and the plain-image information which can improve the security of the following cryptosystem to some extent.

Generally, the size of the plain-image is supposed to be $m \times l$, and the external keys are described with $s$ bytes $(s<m)$. And the external keys can be copped with the original information to make the length be equal to $m$ which will be filled into the $(i+1)_{t h}$ column of the plain-image as shown in Fig. 2. Specifically, the values of pixel in any row are sorted by ascending order to get the corresponding index sequence and find the index number of the first pixel value of this row which is noted the $(i+1)_{t h}$. For instance, the pixel values of the first row of the plain-image with a $6 \times 6$ matrix are 25, 100, 158, 0, 255 and 18 as shown in Fig. 2, and the corresponding sequence with an ascending order is 0 , $18,25,100,158$, and 255 . So the external keys will be filled in the third column because the first pixel value 25 is in the third number. Especially, when the pixel values of the one row are all the same, the external keys are filled into the $(l+1)_{t h}$ column. As a result, a new matrix with the size of $m \times(l+1)$ is obtained to used for the following encryption.

The flow chart of the decimal quantification process is shown in Fig. 3, which includes the following steps.
- Step A1. Divide the plain-image into $m$ rows, and multiply all the elements of this row from left to right to be as A. Specifically, if the $i_{t h}$ value of this row is 0 , it will be replaced by $i \times \pi$. For example, the values of a row are $12,0,15,34,0,255,0$. After the replacement, the novel values are $12,2 \times \pi, 15$, $34,5 \times \pi, 255,7 \times \pi$.

- Step A2. The multiplied value is replaced in terms of the rule of $\mathrm{A}=|\cos (\mathrm{A} \bmod 360)|$, and when it is larger than $10^{14}$, the current value $\mathrm{A}$ is updated. Repeat Step A1.

- Step A3. After the last element of each row is executed, a novel real number (e.g., A) is obtained. And the real number will divide by $10^{14}$, and we can obtain a decimal sequence, e.g., $d_{1}(i), i=1,2$, $\ldots, m$.

- Step A4. Repeat the above steps, and calculate all the elements of a new matrix in the reverse order, i.e., the elements of the matrix are calculated from right to left. Then, another decimal sequence is created, e.g., $d_{2}(i)$.

- Step A5. The sequences $d_{1}(i)$ and $d_{2}(i)$ are continued to be quantified according to Step A1-Step A3, and two decimals $\dot{d}_{1}$ and $\dot{d}_{2}$ are obtained.

- Step A6. Compare the decimal $\dot{d}_{1}$ with $\dot{d}_{2}$, and get the decimal $d$, in that, when $\dot{d}_{1}>\dot{d}_{2}, d$ is equal to $\dot{d}_{2} / \dot{d}_{1}$, Otherwise, $d$ is equal to $\dot{d}_{1} / \dot{d}_{2}$.

As shown in the above steps, the final quantification decimal $d$ has a close relationship with the pixels and external keys. Even if only one bit is changed for the plain-image or external keys, it will cause the decimal $d$ be totally different, which is similar to the avalanche effects of cryptography. Therefore, this quantification method can not only effectively improve the randomness of sequence, but also enhance the security of the encryption system to some extent.

\subsection{The construction of an encryption matrix}

A special encryption matrix is constructed based on the plain-image, external keys and PWLCM chaotic system which will be used for the confusion and diffusion processes of the cryptosystem. Here, the size of a plain-image is supposed to be $m \times n$, and an external key with 32 bytes is arranged in an array with 32 values which is in the range of $(0,255)$. The detailed process of constructing the encryption matrix is shown as follows.

- Step B1. The external key is continued to be quantified as a decimal $d^{\prime}$ according to Step 
A1-Step $A 3$ in Section 2.2 , i.e., the multiplication order is from left to right, and each byte is a multiplier unit.

- Step B2. According to two decimal sequences $d$ (in Section 2.2 and $d^{\prime}$, a new decimal $d^{\prime \prime}$ can be calculated by $d^{\prime \prime}=\left(d+d^{\prime} / 2\right) \bmod 0.5$.

- Step B3. The two decimals $d$ and $d^{\prime \prime}$ are used as the initial value $x(1)$ and control parameter $\mu$ of the PWLCM chaotic system in Eq. 11. Then a corresponding chaotic sequence $\mathbf{x}_{\mathbf{1}}$ is obtained by using $\mathbf{x}_{\mathbf{1}}(i)=\operatorname{PWLCM}\left(d, d^{\prime \prime}\right)$, where $\mathbf{x}_{\mathbf{1}}(i)$ is denoted as $\mathbf{x}_{\mathbf{1}}(i)=\mathbf{x}_{\mathbf{1}}(1), \mathbf{x}_{\mathbf{1}}(2) \cdots, \mathbf{x}_{\mathbf{1}}(200+m)$ and $\mathbf{x}_{\mathbf{1}}(i) \in(0,1)$ with the $10^{-15}$ precision, $i=1,2$, $\cdots, 200+m$.

- Step B4. Similarly, the sequence $\mathbf{x}_{\mathbf{2}}$ can be calculated by $\mathbf{x}_{\mathbf{2}}(i)=\operatorname{PWLCM}\left(d^{\prime} / 2, d^{\prime \prime}\right) / 2$, where $i=1,2, \cdots, 200+m$.

- Step B5. The last $m$ values of the sequences $\mathbf{x}_{\mathbf{1}}$ and $\mathbf{x}_{\mathbf{2}}$ are served as the initial value $x(i)$ and control parameter $\mu$ of the PWLCM chaotic system. Iterate $\mathbf{Y}_{i-200}(j)=\operatorname{PWLCM}\left(\mathbf{x}_{\mathbf{1}}(i), \mathbf{x}_{\mathbf{2}}(i)\right)$ for $n-1$ times, and the $m$ groups of $\mathbf{Y}_{i}$ sequences are obtained, where $i=201,202, \cdots, 200+m$ and $j=1,2, \cdots, n$.

- Step B6. As shown in Fig. 4, realign the $m$ groups of sequences and the $m \times n$ decimal matrix to get the final encryption matrix $\mathbf{K}$.

The encryption matrix $\mathbf{K}$ is implemented in parallel by the PWLCM and the size of which is equal to the size of the plain-image. It effectively increases the security of the encryption system to make the matrix cracking be almost impossible. In addition, cascading the chaotic systems can also avoid the performance degradation of chaos.

\section{The proposed image encryption system}

In this section, a novel parallel image encryption scheme is introduced. There are three stages in the encryption process. First, two quantification decimals are obtained through external keys and plain-image information. They are used as the initial value and control parameter in the PWLCM chaotic system. Then, an encryption matrix and four permutation sequences are constructed based on the PWLCM and FDHCM chaotic system. Finally, a plain-image is permutated and diffused by permutation sequences and the encryption matrix. In addition, the corresponding image decryption process is designed.

A good cryptosystem can not only produce different strong key streams from different plain-images, but also complete self-adoption encryption based on the plain-images. The proposed image encryption system perfectly meets these requirements. We assume that the size of the plain-image $\mathbf{P}$ is $m \times n$, and the external keys are 32 bytes. The encryption process is shown in Fig 5 . The specific implementation process is as follows.

- Step C1. The external keys are filled with plain images, and a new matrix is obtained using the implementation method in Section 2.2 .

- Step C2. Two quantification decimals $d$ and $d^{\prime}$ are obtained based on plain-image information and external keys, as described in Section 2.2 and Section 2.3. They are used as the initial value and control parameter in the PWLCM chaotic system.

- Step C3. Iterate the PWLCM chaotic system $199+x$, where $x=\max (m, n)$ times, a chaotic sequence with the size of $200+x$ is generated, and a matrix $\mathbf{K}$ is reconstructed according to the algorithm in Section 2.3 The matrix $\mathbf{K}$ is quantified using $\mathbf{A}=\left(\mathbf{K} \times 10^{15} \bmod 8\right)+1$ and $\mathbf{B}=\mathbf{K} \times 10^{15} \bmod 256$, in order to ensure that the value of $\mathbf{A}$ and $\mathbf{B}$ are in the range of $(1,8)$ and $(0$, 255), respectively.

- Step C4. After elements from 101 to 104 of the chaotic sequence are used for the FDHCM chaotic system as the initial values, iterate it $x$ times, and the four chaotic sequences $\mathbf{x}_{\mathbf{F} 1}, \mathbf{x}_{\mathbf{F} 2}, \mathbf{x}_{\mathbf{F} 3}, \mathbf{x}_{\mathbf{F} 4}$ are

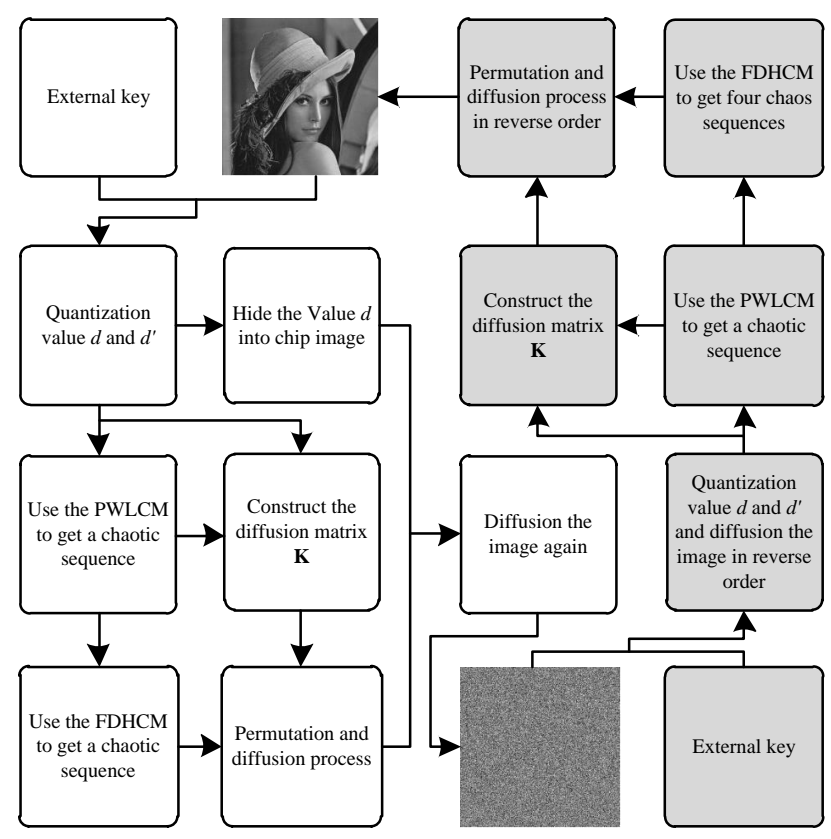

Fig. 5 The image encryption and decryption (in grey) processes. 
generated. Then they are quantified by

$$
\left\{\begin{array}{l}
\mathbf{x}_{\mathbf{F} 1}(i)=\left(\mathbf{x}_{\mathbf{F} 1}(i) \times 10^{15} \bmod n\right)+1 \\
\mathbf{x}_{\mathbf{F} 2}(i)=\left(\mathbf{x}_{\mathbf{F} 2}(i) \times 10^{15} \bmod n\right)+1 \\
\mathbf{x}_{\mathbf{F} 3}(i)=\left(\mathbf{x}_{\mathbf{F 3}}(i) \times 10^{15} \bmod m\right)+1 \\
\mathbf{x}_{\mathbf{F} 4}(i)=\left(\mathbf{x}_{\mathbf{F} 4}(i) \times 10^{15} \bmod m\right)+1
\end{array}\right.
$$

- Step C5. The matrix $\mathbf{A}$ is used to control the pixel of image $\mathbf{P}$ to cyclic shift to the right in the bit-plane by $\mathbf{P}_{\mathbf{1}}(i, j)=B R(\mathbf{P}(i, j), \mathbf{A}(i, j))$, and the new matrix $\mathbf{P}_{\mathbf{1}}$ is obtained, where the $B R$ represents the pixel cyclic shift to right in bit-plane operation, $i=1,2, \cdots, m$ and $j=1,2, \cdots, n$. For example, the value of the image $\mathbf{P}$ and matrix $\mathbf{A}$ in the coordinates $(1,1)$ are 125 and 5 . The right cyclic shift of 125 by five bits is 235 . Therefore, the current value 125 is replaced by 235 .

- Step C6. The sequence $\mathbf{x}_{\mathbf{F} \mathbf{1}}$ is used to control the right cyclic shift of the odd-numbered row in $\mathbf{P}_{\mathbf{1}}$. Conversely, the sequence $\mathbf{x}_{\mathbf{F} 2}$ controls the left cyclic shift of even-numbered row. Similarly, the sequences $\mathbf{x}_{\mathbf{F} 3}$ and $\mathbf{x}_{\mathbf{F} 4}$ control the $\mathbf{P}_{\mathbf{1}}$ odd-numbered line and even-numbered line cyclic shift up and down. Thus the new matrix $\mathbf{P}_{\mathbf{2}}$ is obtained from

$$
\mathbf{P}_{\mathbf{2}}(i, j)= \begin{cases}C R\left(\mathbf{P}_{\mathbf{1}}(i, j), \mathbf{\mathbf { x } _ { \mathbf { F } 1 }}(i)\right) & i \text { is odd } \\ C L\left(\mathbf{P}_{\mathbf{1}}(i, j), \mathbf{\mathbf { x } _ { \mathbf { F } 2 }}(i)\right) & i \text { is even; } \\ C U\left(\mathbf{P}_{\mathbf{1}}(i, j), \mathbf{\mathbf { x } _ { \mathbf { F } 3 }}(j)\right) & j \text { is odd } \\ C D\left(\mathbf{P}_{\mathbf{1}}(i, j), \mathbf{\mathbf { x } _ { \mathbf { F } }}(j)\right) & i \text { is even }\end{cases}
$$

where $C R, C L, C U$, and $C D$ denote the cyclic shift operations in the four vertical directions, respectively, $i=1,2, \cdots, m, j=1,2, \cdots, n$.

- Step C\%. The sequences $\mathbf{x}_{\mathbf{F} 1}$ and $\mathbf{x}_{\mathbf{F} 2}$ are used to control the exchange of the row $\mathbf{P}_{\mathbf{2}}$. If $\mathbf{x}_{\mathbf{F} \mathbf{1}}(i) \neq$ $\left.\mathbf{x}_{\mathbf{F} 2}(i), \mathbf{P}_{\mathbf{2}}(i, j)=E R\left(\mathbf{P}_{\mathbf{2}}\left(\mathbf{x}_{\mathbf{F} 1}(i), j\right)\right), \mathbf{P}_{\mathbf{2}}\left(\mathbf{x}_{\mathbf{F} 2}(i), j\right)\right)$. In the same way, sequences $\mathbf{x}_{\mathbf{F} 3}$ and $\mathbf{x}_{\mathbf{F} 4}$ control the exchange of the column $\mathbf{P}_{\mathbf{2}}$. If $\mathbf{x}_{\mathbf{F} \mathbf{3}}(j) \neq \mathbf{x}_{\mathbf{F} \mathbf{4}}(j)$, $\mathbf{P}_{\mathbf{2}}(i, j)=E C\left(\mathbf{P}_{\mathbf{2}}\left(i, \mathbf{x}_{\mathbf{F} 3}(j)\right), \mathbf{P}_{\mathbf{2}}\left(i, \mathbf{x}_{\mathbf{F} 4}(j)\right)\right)$. The $E R$ and $E C$ represent the exchange operations for the row and column, respectively, and $i=1,2, \cdots$, $m, j=1,2, \cdots, n$.

- Step C8. The image $\mathbf{P}_{\mathbf{2}}$ is diffused by matrix $\mathbf{B}$. The encryption image $\mathbf{E}$ is obtained by $\mathbf{E}(i, j)=((\mathbf{P 2}(i, j)+\mathbf{B}(i, j)) \bmod 256) \oplus \mathbf{B}(i, j)$, where $\oplus$ represents the bitxor operation, $i=1,2$, $\cdots, m$ and $j=1,2, \cdots, n$.

- Step C9. The decimal $d$ is hidden from the encryption image $\mathbf{E}$. For example, if the decimal $d$ is 0.354864172356179 , and the first pixel in the encryption image $\mathbf{E}$ is $v$. The decimal $d$ is divided into eight integers $39,57,41,86,65,43,12$, and 7 . They will replace the eight pixels, which are the coordinates between $(v, n)$ and $(v, n-7)$ in encryption image $\mathbf{E}$. If $v>n$, the new line number $t$ is obtained by $t=v \bmod n$, and the eight pixels of coordinates between $(t, n)$ and $(t, n-7)$ are replaced.

- Step C10. In addition to the above steps, the encryption image $\mathbf{E}$ is applied by an extra round of XOR operation in the bit-plane. This can further enhance cryptosystem security and obtain a uniform distribution of encrypted images to defend against statistical attack and differential attack. The operation process is given by

$$
\left\{\begin{array}{c}
\mathbf{E}(i+1,1)=\mathbf{E}(i, 1) \oplus \mathbf{E}(i+1,1) ; \\
\mathbf{E}(i+1,2)=\mathbf{E}(i, 2) \oplus \mathbf{E}(i+1,2) ; \\
\vdots \\
\mathbf{E}(i+1, n)=\mathbf{E}(i, n) \oplus \mathbf{E}(i+1, n) ; \\
\mathbf{E}(1, j+1)=\mathbf{E}(1, j) \oplus \mathbf{E}(1, j+1) ; \\
\mathbf{E}(2, j+1)=\mathbf{E}(2, j) \oplus \mathbf{E}(2, j+1) ; \\
\vdots \\
\mathbf{E}(m, j+1)=\mathbf{E}(m, j) \oplus \mathbf{E}(m, j+1),
\end{array}\right.
$$

where $i=1,2, \cdots, m$ and $j=1,2, \cdots, n$.

Now the encryption image $\mathbf{E}$ is obtained and the quantification decimal is randomly hidden in $\mathbf{E}$. According to symmetric encryption principles, the process of decryption is an inverse process, as shown in Fig. 5. The details are given by the following steps.

- Step D1. First, a round of inverse XOR-operation in terms of the above Step C10 is performed.

- Step D2. According to the first pixel in the encryption image, the location of the decimal replaced is locked, and the eight integers in the cipher-image are taken out. For example, they are $39,57,41,86,65,43,12$, and 7 , and then, the decimal $d$ is reconstituted by $d=3 \times 10^{-1}+5 \times$ $10^{-2}+\cdots+7 \times 10^{-8}+\cdots+7 \times 10^{-14}+9 \times 10^{-15}$.

- Step D3. The decimal $d^{\prime}$ and the encryption matrices $\mathbf{A}$ and $\mathbf{B}$ are reconfigured in the same way of Section 2.2 and 2.3 Then, the four chaotic sequences $\mathbf{x}_{\mathbf{F} 1}, \mathbf{x}_{\mathbf{F 2}}, \mathbf{x}_{\mathbf{F 3}}, \mathbf{x}_{\mathbf{F} 4}$ are reconstructed as described in the above Step $C 4$.

- Step D4. In terms of the sequences $\mathbf{x}_{\mathbf{F} 1}, \mathbf{x}_{\mathbf{F} 2}, \mathbf{x}_{\mathbf{F} 3}$, $\mathbf{x}_{\mathbf{F} 4}$ and encryption matrixes $\mathbf{A}$ and $\mathbf{B}$, the encryption image is executed by the processes of permutation and diffusion in reverse order, and the decrypted image is obtained.

\section{Algorithm implemented on the GPU}

In this section, the parallel image encryption algorithm is designed and implemented on a GPU 
device. The proposed algorithm consists of four stages: the generation of quantification decimal, the construction of encryption matrix, the process of image permutation and the process of diffusion. If the four stages can be processed in parallel, the execution time can be reduced, which ensures the security of image encryption and in the meantime improves the efficiency of the algorithm. The four stages of the proposed algorithm have a high degree of parallelism, thus it is possible to implement it in parallel. Fig. 6 shows the flow chart of the image encryption algorithm on the GPU.

The corresponding kernel parameters are given in Table 1, and the related symbol descriptions are shown in Table 2. The first kernel is the process of obtaining two quantification decimals, and the concrete implementation method is as shown in Algorithm 1. In Algorithm 1, the function $\mathrm{M}()$ represents external keys filled in the plain-image and the function $\mathrm{Q}()$ is the parallel quantification process. We set the NDRange as one-dimensional. The global work size and local work size parameters are $[m \times n$, $0,0]$ and $[1,0,0]$. The encryption matrix can be obtained by using the second kernel, and the kernel pseudo-code is as shown in Algorithm 2. We use the inputs of the quantification decimals $d$ and $d^{\prime}$ and the image size $m$ and $n$. Two encryption matrices $\mathbf{A}$ and $\mathbf{B}$ are outputted, which are used in the permutation and diffusion processes. Algorithm 3 shows the process of permutation. From pseudo-code Line 2 to 6 , the encryption matrix $\mathbf{A}$ is used to control the plain-image pixel cycle shift in the bit-plane. The different rows and columns of the image are cyclic shifted by the chaotic sequence $\mathbf{x}_{\mathbf{F} 1}, \mathbf{x}_{\mathbf{F} 2}, \mathbf{x}_{\mathbf{F} 3}, \mathbf{x}_{\mathbf{F} 4}$, as shown by Line 7 to 20 of Algorithm 3 . Then the image pixel of different rows and columns are exchanged as Line 21 to 30 shows. Finally, Algorithm 4 represents

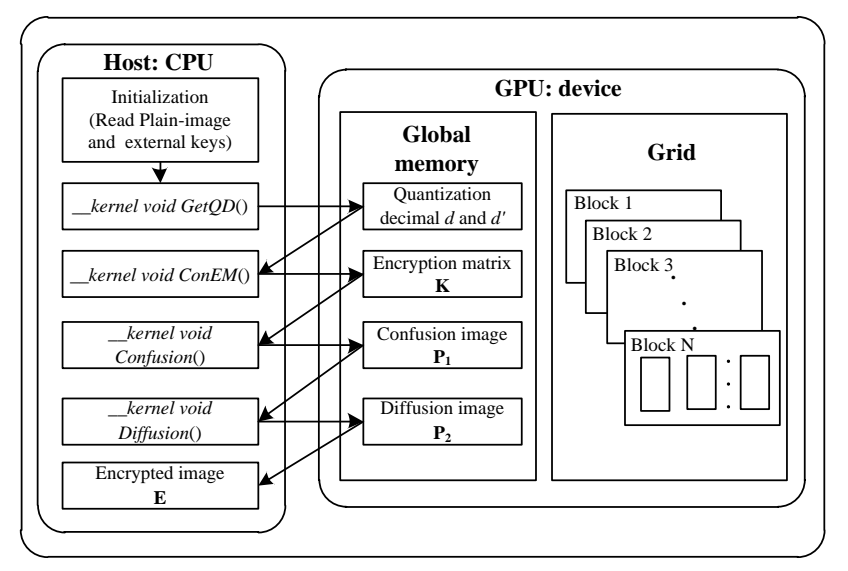

Fig. 6 The parallel image encryption mechanism. the fourth kernel method, which is the diffusion process of image encryption. The permutated image $\mathbf{P}_{\mathbf{1}}$ and encryption matrix $\mathbf{B}$ are executed by the bitxor operation (see Line 3 to 5 of Algorithm 4), then the encryption image $\mathbf{E}$ is finally obtained through a new round of bitxor operation in the bit-plane, as shown by Line 6 to 11 of Algorithm 4
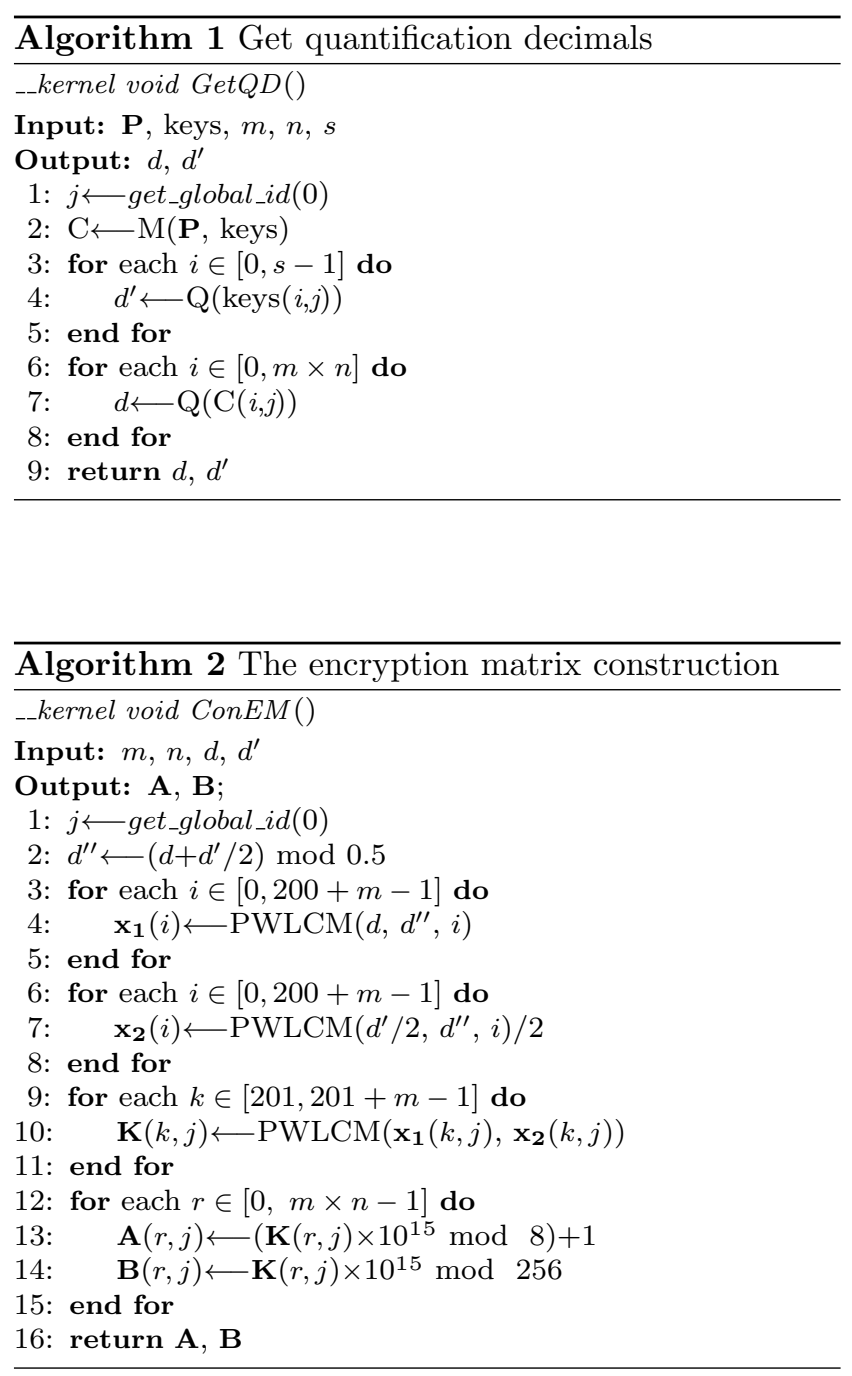

\section{Simulations and security analysis}

This section gives the simulation results of the proposed scheme. The encryption and decryption programs are executed by Microsoft Visual $\mathrm{C}++2010$ and Windows 7 operating system. The four parameters of the FDHCM are $a=10, b=3 / 8, c=28$ and $d=-1$. The external keys are $184,52,1,249,252$, 177, 99, 209, 256, 24, 61, 102, 57, 240, 49, 249, 113, 29, 154, 196, 101, 64, 199, 5, 133, 103, 45, 197, 123, 243, 
Table 1 The parameter configurations of four kernels.

\begin{tabular}{|c|c|c|c|c|c|}
\hline & NDRange & global_work_size & local_work_size & Input & Output \\
\hline _-kernel void GetQD() & $1-\mathrm{D}$ & {$[m \times n, 0,0]$} & {$[1,0,0]$} & $\mathbf{P}$, keys, $m, n, s$ & $d, d^{\prime}$ \\
\hline __kernel void ConEM() & $1-\mathrm{D}$ & {$[m \times n, 0,0]$} & {$[1,0,0]$} & $d, d^{\prime}, m, n$ & $\mathbf{A}, \mathbf{B}$ \\
\hline _-_kernel void Confusion() & $1-\mathrm{D}$ & {$[\Delta, 0,0]$} & {$[\Delta, 0,0]$} & $\mathbf{P}, \mathbf{A}, \mathbf{x}_{\mathbf{F} 1}, \mathbf{x}_{\mathbf{F} \mathbf{2}}, \mathbf{x}_{\mathbf{F} \mathbf{3}}, \mathbf{x}_{\mathbf{F} \mathbf{4}}, m, n$ & $\mathbf{P}_{2}$ \\
\hline __kernel void Diffusion() & $2-\mathrm{D}$ & {$[m, n, 0]$} & {$[16,16,0]$} & $\mathbf{P}_{1}, \mathbf{B}, m, n$ & $\mathbf{E}$ \\
\hline
\end{tabular}

Table 2 Related symbol descriptions.

\begin{tabular}{lc}
\hline $\mathrm{P}$ & Plain-image \\
\hline$m, n$ & The size (length, width) of the image \\
keys & External keys \\
$s$ & The size of the external keys \\
$\Delta$ & $\Delta=\max (m, n)$ \\
$\mathbf{P}_{\mathbf{1}}, \mathbf{P}_{\mathbf{2}}$ & The confusion and diffusion image \\
$d, d^{\prime}$ & Quantification decimals \\
$\mathbf{A}, \mathbf{B}$ & Encryption matrices \\
$\mathbf{\mathbf { X } _ { \mathbf { F } 1 }}, \mathbf{x}_{\mathbf{F} \mathbf{2}}, \mathbf{x}_{\mathbf{F} 3}, \mathbf{x}_{\mathbf{F} 4}$ & Confusion sequences (in Section 3$)$ \\
$\mathbf{E}$ & Encrypted image \\
\hline
\end{tabular}

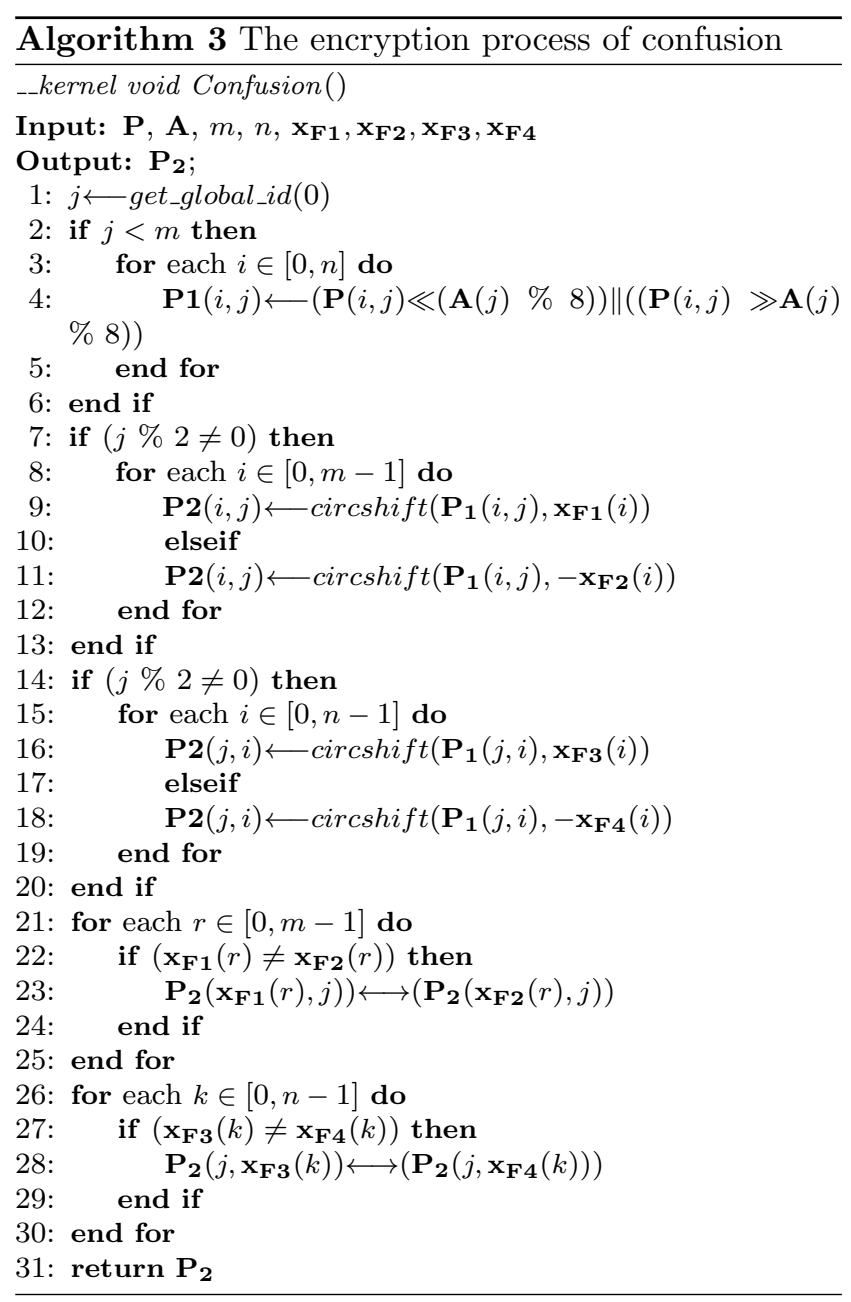

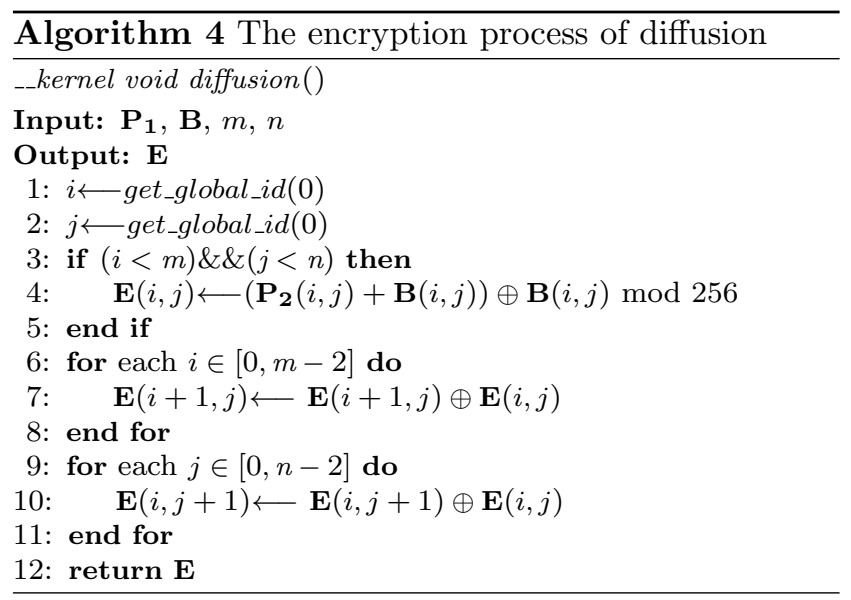

33, and 75 (32 Bytes) in decimal. A number of experiments were performed based on several images, which were used as plain-images with the sizes of $128 \times 128,256 \times 256,512 \times 512$ and $1024 \times 1024$. In Fig. 7 and Fig. 8, we show the encryption and decryption results of the image of Lena with of the size of $512 \times 512$, which were executed on the CPU and GPU devices, respectively. In the following subsections, several tests are discussed to verify the security of the proposed cryptosystem.
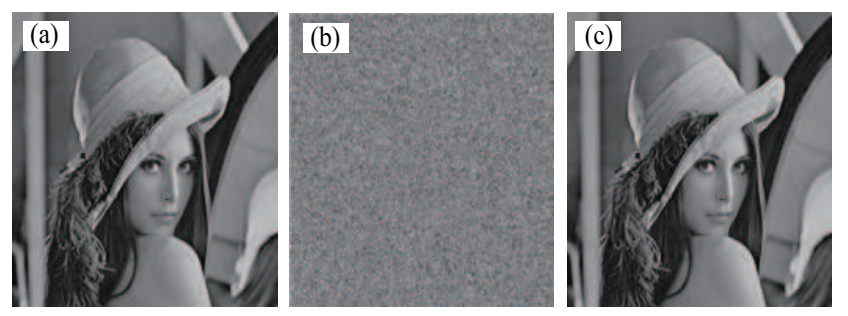

Fig. 7 Encryption and decryption results on a CPU device. (a) Original image; (b) Encryption image; (c) Decryption image.

\subsection{Encryption efficiency analysis}

To ensure real-time data transmission, the image encryption algorithm should guarantee the security of 

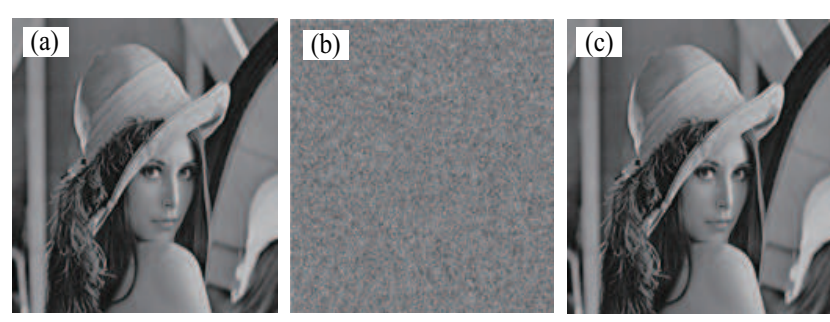

Fig. 8 Encryption and decryption results on a GPU device. (a) Original image; (b) Encryption image; (c) Decryption image.

Table 3 The encryption time of the different size images on CPU and GPU devices.

\begin{tabular}{lccc}
\hline \multirow{2}{*}{ Image size } & \multicolumn{2}{c}{ Time $(m s)$} & \multirow{2}{*}{ Speed-up ratio $(r)$} \\
\cline { 2 - 3 } & CPU & GPU & \\
\hline \multirow{2}{*}{$128 \times 128$} & 6.1239 & 0.7120 & 8.6010 \\
$256 \times 256$ & 25.4828 & 1.8216 & 13.9892 \\
$512 \times 512$ & 92.6924 & 3.4365 & 26.9729 \\
$1024 \times 1024$ & 396.5155 & 6.9153 & 57.3389 \\
\hline
\end{tabular}

transmission and also have a fast speed to encrypt the image. The proposed parallel image encryption scheme is processed on the CPU and GPU devices, and the speed-up ratio $r$ is calculated, which can be defined by $r=T_{s} / T_{p}$, where $T_{s}$ and $T_{p}$ are the executed time on the CPU and GPU, respectively. We use four different image sizes to compare the encryption speeds of both the devices. The experimental results are shown in Table 3 .

From Table 3 we can see that regardless of the used devices (GPU or CPU), if the image size increases, the processing time is longer, but the speed-up ratio is also greater. The time spent on the GPU is less than CPU. For example if the image size is $128 \times 128$, the speed-up ratio $r$ is 8.6. However, when the image size is $1024 \times 1024$, the $r$ increases to 57.3. Thus the proposed algorithm processed the larger size image on the GPU more efficiently than on the CPU, which also indicates that the execution of the encryption algorithm is improved using the parallel computing via a GPU device.

\subsection{Key space analysis}

The key space of an encryption algorithm is the set of different keys that can be used for encryption. The key space should be sufficiently large to defend against brute-force attack. In the proposed cryptosystem, the keys are 1$)$. the parameter $\mu$ and the initial value $x(0)$ of the PWLCM; 2). the parameters $a, b, c, d$ and the initial values $\mathbf{x}_{\mathbf{F} 1}(1), \mathbf{x}_{\mathbf{F} 2}(1), \mathbf{x}_{\mathbf{F} 3}(1), \mathbf{x}_{\mathbf{F} 4}(1)$ of the
FDHCM; and 3). the 256-bit external keys. For the parameters and initial values of the PWLCM and FDHCM, if the precision is $10^{15}$, the key space size is $10^{150}$. The size of the external keys is 256-bit, which means that the key space is $2^{256}$. Therefore, the total key space is $S=10^{150} \times 2^{256}$. In addition, the different plain-images generate exclusive initial values and control parameters. The key space is probably greater than $10^{150} \times 2^{256}$. In this case, such a large key space can guarantee security against brute-force attacks.

\subsection{Differential attack}

To resist a differential attack, a secure cryptosystem should be sensitive to the plain-image. Small changes (e.g., one bit) in the plain-image must create a large difference in the encrypted image. Differences can be measured by two criteria, i.e. the number of pixel change rate $(\mathrm{NPCR})$ and the unified average changing intensity (UACI). The former is used to measure the number of different pixels between two images, and the latter is used to measure the average intensity difference. The NPCR and UACI are defined by

$$
N P C R=\frac{\sum_{i, j} D(i, j)}{N} \times 100 \%
$$

and

$$
U A C I=\frac{1}{N}\left[\sum_{i, j} \frac{\left|E_{1}(i, j)-E_{2}(i, j)\right|}{2^{n}-1}\right] \times 100 \%,
$$

where $N$ is the total number of pixels in the image, $n$ is an n-bit greyscale, and $E_{1}$ and $E_{2}$ are the two cipher-images whose corresponding plain-images have only a one-bit difference. The difference array $\mathrm{D}(i, j)$ is determined by the following rule: when pixels $(i, j)$ in $E_{1}$ and $E_{2}$ are different (i.e., $E_{1}(i, j) \neq E_{2}(i, j)$ ), then $\mathrm{D}(i, j)=1$; otherwise, $\mathrm{D}(i, j)$ is 0 . The probability distribution of $\mathrm{D}(i, j)$ is given by

$$
D(i, j)=\left\{\begin{array}{l}
0, C_{1}(i, j)=C_{2}(i, j), p_{0}=\frac{1}{2^{n}} \\
1, C_{1}(i, j) \neq C_{2}(i, j), p_{1}=1-\frac{1}{2^{n}} .
\end{array}\right.
$$

In addition, the expected values of the NPCR and UACI for a strong encryption scheme are given by

$\mathrm{NPCR}_{\mathrm{e}}=1-\frac{1}{2^{n}}$

and

$\mathrm{UACI}_{\mathrm{e}}=\frac{1}{N}\left[\frac{2 \sum_{i=1}^{2^{n}-1} i \times\left(2^{n}-i\right)}{2^{n}-1}\right]$, 
respectively. For an 8-bit grey scale image, the $\mathrm{NPCR}_{\mathrm{e}}$ and $\mathrm{UACI}_{\mathrm{e}}$ are $99.6094 \%$ and $33.4635 \%$. In this approach, the quantitative measures of NPCR and UACI are calculated for five different plain-images, and their results are shown in Table 4, The tested pixels are all at $(1,1)$ in each image.

In order to further verify the sensitivity of the plain-image, all pixels in the image with the size of $256 \times 256$ are selected, and each pixels lowest bit is changed to obtain 65536 corresponding cipher-images. Comparing these 65536 cipher-images with the original encrypted image, we can obtain the NPCR and UACI. The results are shown in Fig. 9. The average NPCR and UACI for these 65536 tests are $99.6203 \%$ and $33.4805 \%$, which are better than the expected values. It indicates that the proposed encryption scheme is very sensitive to the small changes in the plain-image and that the rate of influence related to single-pixel change in the plain-image is very high. Therefore, the proposed encryption algorithm is robust against differential attack.

Table 4 The NPCR and UACI for different plain-images.

\begin{tabular}{llllll}
\hline Images & Lena & Baboon & Peppers & Plane & Elaine \\
\hline NPCR(\%) & 99.6399 & 99.6132 & 99.6244 & 99.6124 & 99.6052 \\
UACI(\%) & 33.6910 & 33.4354 & 33.4349 & 33.4085 & 33.5370 \\
\hline
\end{tabular}
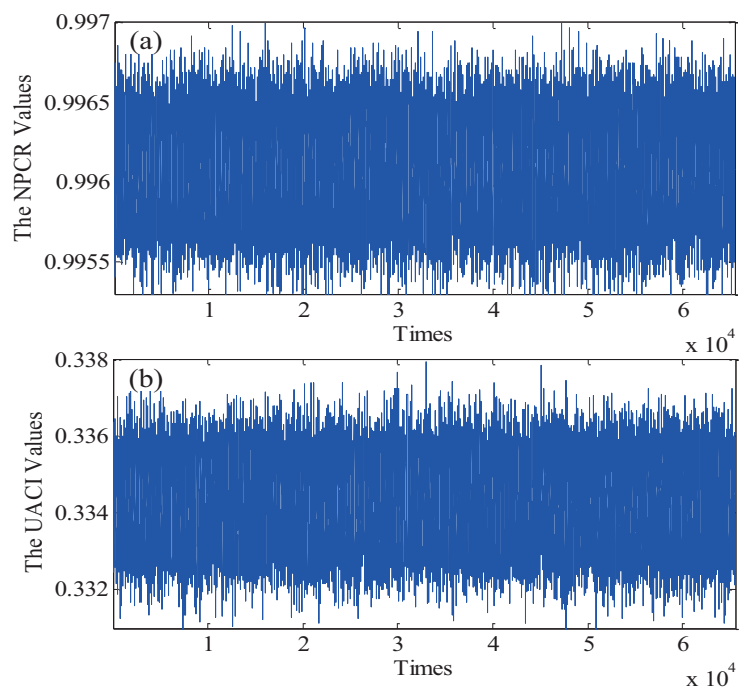

Fig. 9 The results of differential attack. (a) NPCRs for all modified plain images; (b) UACIs for all modified plain images.

\subsection{Key sensitivity analysis}

Key sensitivity is an essential feature for any secure algorithm that can resist brute-force attack. Several key sensitivity tests are performed in this approach. Fig. 10(b) shows the encrypted image of Lena with the correct encryption keys. The FDHCM parameters are $a=10, b=3 / 8, c=28$, and $d=-1$, and the external keys are 184, 52, 1, 249, 252, 177, 99, 209, 256, 24, 61, $102,57,240,49,249,113,29,154,196,101,64,199,5$, 133, 103, 45, 197, 123, 243, 33, and 75. Firstly, the parameters of the FDHCM are fixed, while the input external keys are changed by only one bit. The cipher-image is shown in Fig. 10(c). Then, the external keys are fixed, while the FDHCM parameters change by one bit, e.g., $a=10+10^{-14}, b=3 / 8+10^{-14}$, $c=128+10^{-14}, \quad d=-1+10^{-14}$. We obtain their cipher-images as shown in Fig. 10(d) and (g). Comparing them with the original cipher-image shown in Fig. 10(b), the five differential charts are obtained as shown in Fig. 10(h)-(l). Fig. 10(m)-(q) are the histograms of Fig. 10(h)-(l), which show that there are huge differences between the original cipher-image and Fig. 10(c)-(g). The NPCR values of the five experiments are 99.62\%, 99.64\%, 99.61\%, 99.63\% and $99.61 \%$. As the previous test results indicate, a tiny difference in the key results in major changes in the corresponding cipher-images. Therefore, the proposed encryption scheme has high key sensitivity.

\subsection{Statistical analysis}

A histogram shows the distribution of pixels in an image. The encrypted image of a good cryptosystem should have a uniform histogram to resist any statistical attacks. The histograms of the original five plain-images and their corresponding encrypted images are shown in Fig. 11. We can see that the distributions of all cipher-images appear to be uniform regardless of how their original images are related. The adjacent pixels of the original image have a high correlation in the horizontal, vertical and diagonal directions. An ideal encryption algorithm can make the correlation coefficients of the pixels in the encrypted image be sufficiently low to resist statistical attacks. To analyse and compare the correlations of the adjacent pixels in the plain- and cipher-image, the image Lena was used as an example. We randomly choose $10^{14}$ pairs of adjacent pixels in the horizontal, vertical and diagonal directions from the plain-image and its encrypted image. The correlation distributions of two adjacent pixels in three directions are shown in Fig. 12 , 

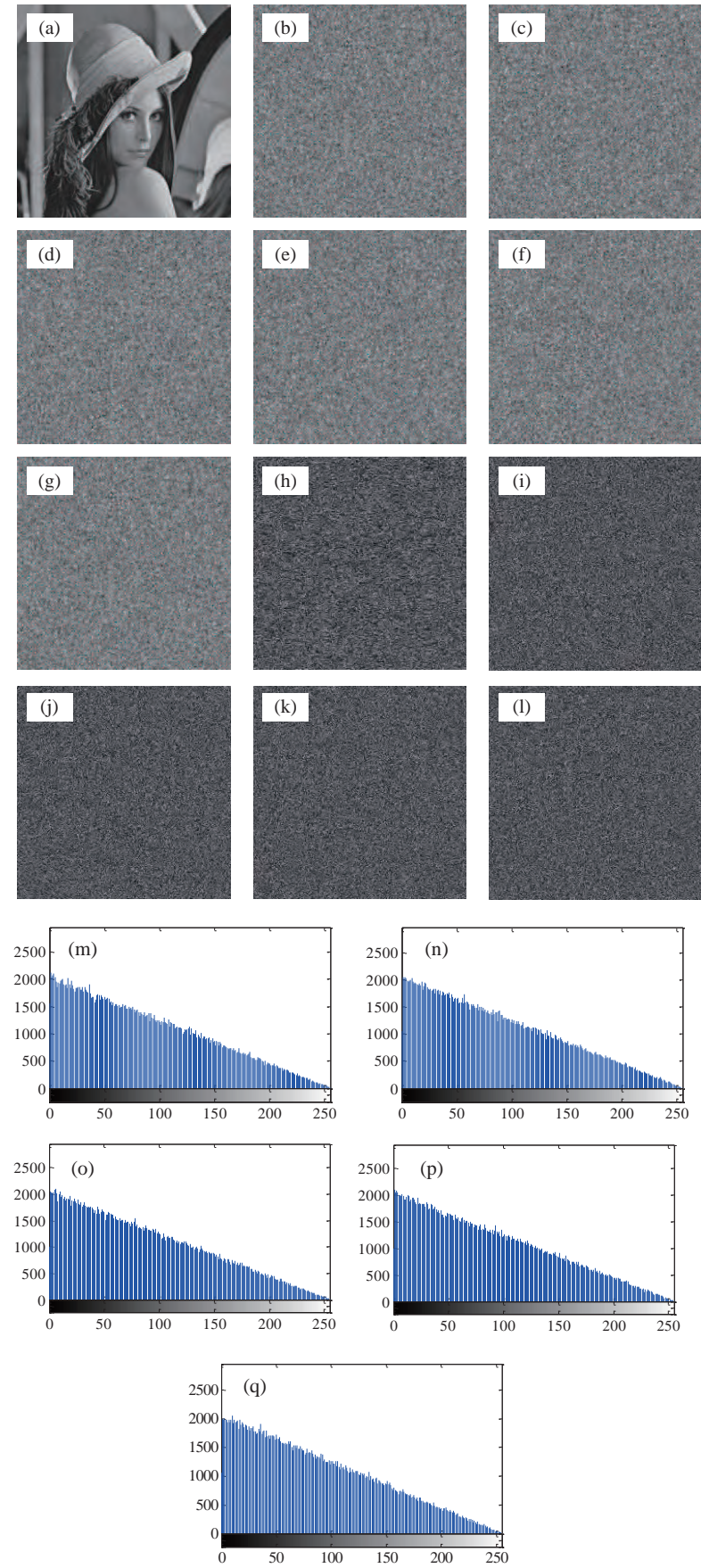

Fig. 10 The results of key sensitivity analysis. (a) is the plain-image Lena. (b) is the cipher-image with the correct encryption keys. (c) is the cipher-image with a slight change in the external key. (d)-(g) are cipher-images with a slight change in the FDHCM parameters. (h)-(l) are the differences between original cipher-image and $(\mathrm{d})-(\mathrm{g}),(\mathrm{m})-(\mathrm{q})$ are the grey histograms of (h)-(l).
It is obvious that the plain-image has a strong correlation among adjacent pixels. However, the correlation in the corresponding cipher-image is largely weakened. We calculate the correlation coefficient $r_{x y}$ of each pair by

$$
r_{x y}=\frac{\operatorname{cov}(x, y)}{\sqrt{D(x) D(y)}},
$$

where

$\operatorname{cov}(x, y)=\frac{1}{N} \sum_{i=1}^{N}\left(x_{i}-E(x)\right)\left(y_{i}-E(y)\right)$,

$D(X)=\frac{1}{N} \sum_{i=1}^{N}\left(x_{i}-E(x)\right)^{2}$,

$E(X)=\frac{1}{N} \sum_{i=1}^{N} x_{i}$

$x$ and $y$ are the greyscale values of two adjacent pixels in the image, and $N$ is the total number of pixels selected from the image.
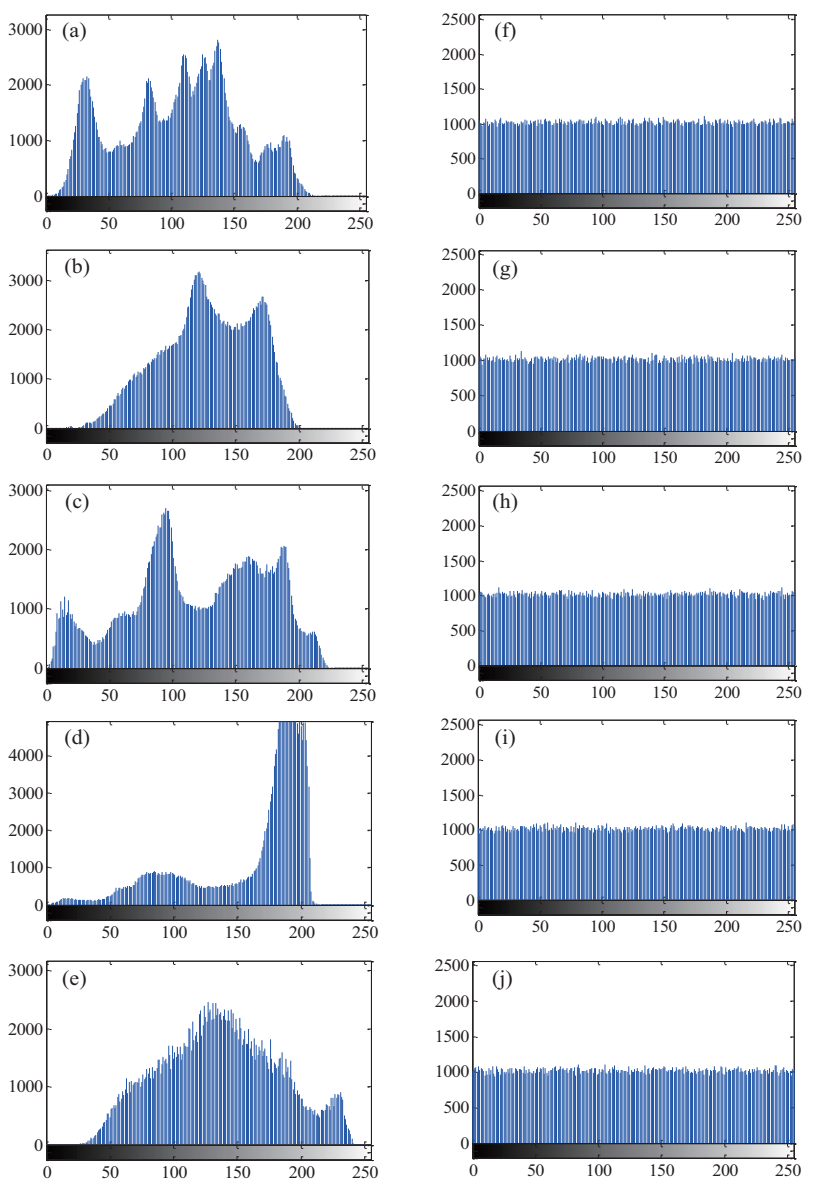

Fig. 11 The histogram of the five different plain- and chiper- images. (a)-(e) The histograms of plain-images Lena, Baboon, Pepper, Plane and Elaine; (f)-(j) The histograms of corresponding cipher-images. 
Table 5 Correlation coefficients of plain- and cipher-images.

\begin{tabular}{|c|c|c|c|}
\hline \multirow{3}{*}{ Images - } & \multicolumn{3}{|c|}{ Correlation coefficients in } \\
\hline & Horizontal & Vertical & Diagonal \\
\hline & Cipher & Cipher & Cipher \\
\hline Lena & 0.97680 .0073 & 0.96670 .0090 & $0.9493-0.0053$ \\
\hline Baboon & $0.8627-0.0019$ & 0.77190 .0074 & 0.72450 .0005 \\
\hline Pepper & 0.96340 .0092 & 0.97550 .0068 & $0.9670-0.0084$ \\
\hline Plane & $0.9671-0.0079$ & 0.96490 .0014 & $0.9354-0.0015$ \\
\hline Elaine & $0.9743-0.0025$ & $0.9731-0.0021$ & 0.96230 .0063 \\
\hline
\end{tabular}

The calculation results are listed in Table 5. These results clearly show that the correlation coefficients of the plain-image are close to 1 , while those of the cipher-image are nearly 0 . There is no correlation between neighbouring pixels in the cipher-image. From Table 5. we can see that the proposed algorithm has good characteristics for withstanding statistic attacks.
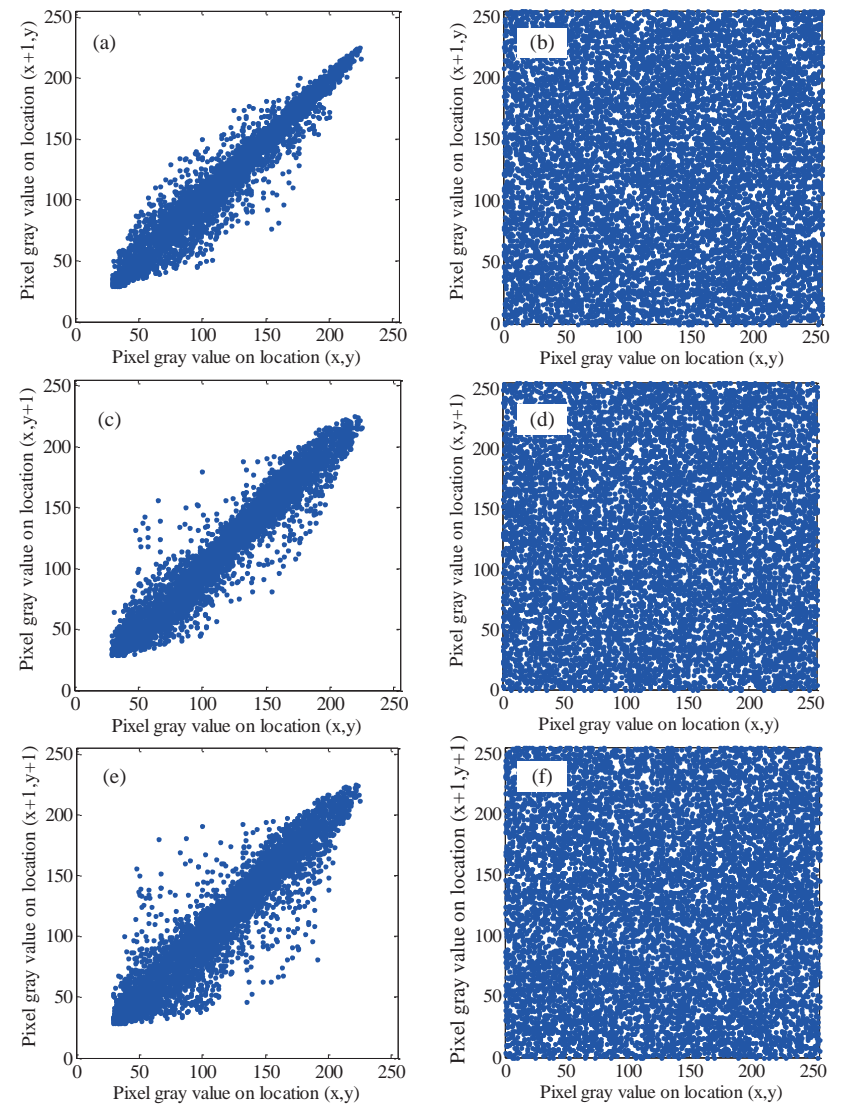

Fig. 12 Distributions of two adjacent pixels in the plainimage and in the cipher-image. (a) horizontal correlation of original image, (b) horizontal correlation of cipher image, (c) vertical correlation of original image, (d) vertical correlation of cipher image, (e) diagonal correlation of original image, (f) diagonal correlation of cipher image.
5.6 Entropy analysis

Information entropy is the most important measure of randomness. The source of information is defined as $m$; we can use

$$
H(m)=\sum_{i=0}^{2^{n}-1} p\left(m_{i}\right) \log _{2} \frac{1}{p\left(m_{i}\right)}
$$

to calculate information entropy, where $p\left(m_{i}\right)$ is the probability of the $m_{i}$. Assume that there are $2^{8}$ states of the symbols and that they appear with the same probability. According to Eq. (7), we can obtain the ideal $H(m)=8$, which shows that the information is random. Therefore, for a ciphered image with 256 grey levels, the entropy should ideally be close to 8 . The closer it is to 8 , the lower the probability for the cryptosystem to divulge any information. We use Eq. (7) to calculate the information entropy of the five cipher-images. The entropy values of the five cipher-images are shown in Table 6, and they are all close to the ideal value of 8 , which indicates that the proposed algorithm has a good property of information entropy.

Table 6 The entropies of different cipher-images.

\begin{tabular}{llllll}
\hline Images & Lena & Baboon & Pepper & Plane & Elaine \\
\hline Entropy & 7.999382 & 7.999280 & 7.999283 & 7.999344 & 7.999376 \\
\hline
\end{tabular}

\subsection{Chosen/known plain-image attack}

In this paper, we consider the following three points to effectively avoid the known-plaintext and chosen-plaintext attacks: (a) The construction of ergodic sequences is based on the initial key and different plain-images, and they are produced by PWLCM and FDHCM chaotic systems. When the plain-image is changed, there are different initial values and system parameters. Even for the same original image, the initial values and the system parameters are totally different if the image has a small change; (b) The construction of the encryption matrix is based on PWLCM chaotic systems. It is generated in parallel with the size $m \times n$. The possible existing different permutations could achieve $m ! \times n$, which shows a strong robustness; and (c) The permutation and diffusion processes can occur at the same time. Therefore, our algorithm has high dependency on the plain-image and is secure against known-plaintext and chosen-plaintext attacks 16, 46. 
For plain-image attacks, the cryptanalyst tries to find the secret key by using all black or all white images as special plain-images to attack the encryption algorithms. Special images can make the encryption algorithm unsafe, and the keys are obtained by analysing the encrypted image if the cryptosystem is relatively simple. However, it cannot decrypt other cipher-images using the same keys because the keys of our cryptosystem are strictly closed to the plain-image information. Different plain-images will produce totally different secret keys. Therefore, our encryption algorithm can effectively resist the known-plaintext and chosen-plaintext attacks.

\subsection{Occlusion and noise attack analysis}

During the transmission of cipher-images via the networks, they may be cropped or influenced by noise; or some data may be lost because of a congested network or malicious destruction. Therefore, it should be determined if an encryption scheme is able to resist cropping and noise attacks 45. If the damaged cipher-image can be recovered successfully, the encryption scheme has the ability to defend against occlusion and noise attacks $3,5,34$. Firstly, we test the ability of our encryption scheme to resist an occlusion attack. Fig. 13(a)-(d) show the experimental results of data loss attacks on the cipher-image of Fig. 7(b) with different occlusions, and Fig. 13(e)-(h) show the recovered images. From the results, we can see that when the cipher-images have $12.5 \%, 25 \%$, $50 \%, 75 \%$ occlusion, the image information can still be recognized even though a strong occlusion attack has occurred. This achieves a better performance than the encryption schemes in [5, 36, which fails to complete. It is proven that our scheme is immune against occlusion attack. For example, if less than $25 \%$ of the cipher data is lost, the resolution of the recovered images shown in Fig. 13(e)-(f) contains almost all visual information of the plain-image. Even though $75 \%$ of the cipher-image shown in Fig. 13(d) is occluded, the decrypted image can still be recognized, as shown in Fig. 13(h). Therefore, the proposed method is capable of resisting occlusion attack.

The salt and pepper noise and Gaussian attack are evaluated here since they are two typical noises. Fig. 14(a)-(c) are the encrypted Lena images, which are influenced by the salt and pepper noise with $5 \%$, $10 \%$, and $20 \%$ densities. Fig. 14(d)-(f) are the decryption of Fig. 14(a)-(c) and show that the encrypted image influenced by the salt and pepper noises can be decrypted correctly. Fig. 15 illustrates the cipher-image of Fig. 7(b) affected by Gaussian noise with different variances and the corresponding decrypted image. From Fig. 15(c)-(f), we can see that when the variances of the noise are $0.0001,0.0003$ and 0.0005 , the image information can be clearly recognized. Compared with [3, we find that under the same conditions, the performance of the proposed algorithm in this approach is much better. Therefore, the proposed method has the capability to resist occlusion and noise attacks.

\subsection{Speed analysis and comparisons}

In addition to security evaluations for image cryptosystem schemes, the execution time is a very important factor, especially for real time multimedia applications. To evaluate the proposed scheme, the standard image Lena with a size of $512 \times 512$ is selected. The hardware environment is based on an Intel Core i7 CPU, an NVIDIA GeForce GTX $750 \mathrm{Ti}$ GPU, and 8 GB memory. The test is executed 500 times. The mean test time of every step is listed in Table 7. As Fig. 16 shows, the quantification process and the construction of the encryption matrix occupy $15.1255 \%$ and $11.1302 \%$ of the total time, and the pixel permutation and diffusion process takes up $54.1527 \%$ of the total time. Compared with the approaches of 11, 15, 30, the proposed encryption method has higher security because its quantification design is more complex and effective. The quantified decimal is obtained through a novel parallel scheme, and the speed is faster than Ref [27]. The initial values of chaotic map are strictly related to plain-image information.

Comparisons with other algorithms are listed in Table 8, which includes information entropy, key space, NPCR and UACI values, and execution time. As seen from Table 8 , the entropy value of the proposed algorithm is better than that of other schemes. In terms of the key space, it should be larger than $2^{100}$ to defend from a brute force attack $[9$. The key space of this work is larger than Ref [1, 8, 31, 44, 47, but smaller than the scheme in 10]. However the NPCR and UACI values in Ref [10] are smaller. In addition, the proposed algorithm is faster than [1, 10, 31, 44, 47], but slower than 8. Although the algorithm in the approach of 8 only takes $3.41 \mathrm{~ms}$ for encryption, however this time is only for one round encryption. The histograms showed that the algorithm in 8 is not safe if using only one round encryption, therefore it normally needs more than six rounds to get a good security performance which takes a long time to complete all these steps. 

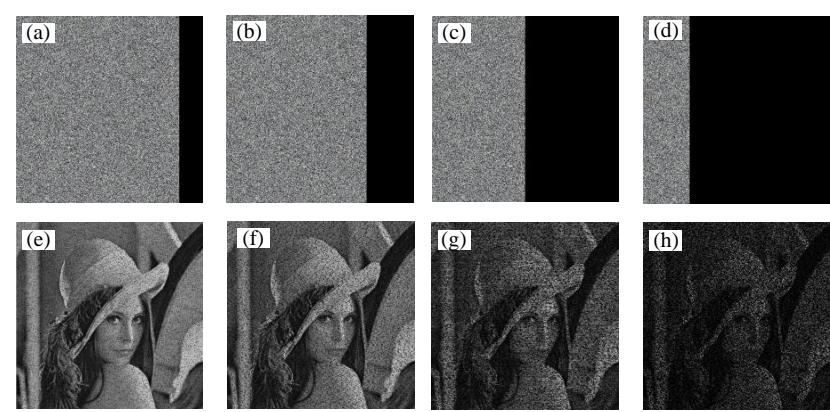

Fig. 13 The decryption images under different occlusion attacks. (a) the cipher-image have $12.5 \%$ occlusion, (b) the decryption image of $25 \%$ occlusion, (c) the cipher-image have $50 \%$ occlusion, (d) the decryption image of $75 \%$ occlusion, (e) the cipher-image have $50 \%$ occlusion, (f) the decryption image of $50 \%$ occlusion, (g) the cipher-image have $75 \%$ occlusion, (h) the decryption image of $75 \%$ occlusion.
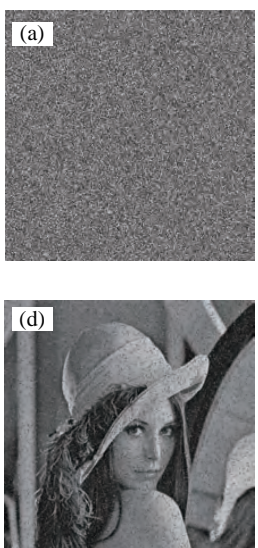
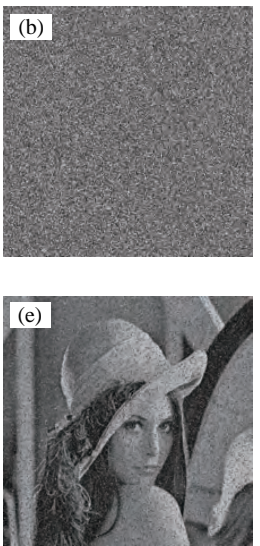
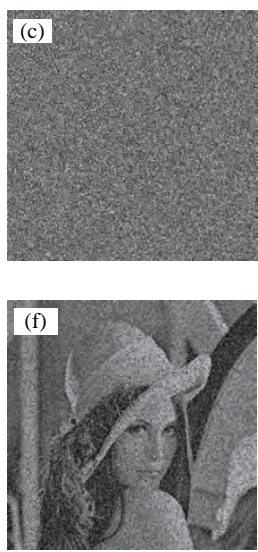

Fig. 14 Cipher- and decrypted images under salt and pepper noises with different levels. (a) Noise density $=5 \%$; (b) Noise density $=10 \%$; (c) Noise density $=20 \%$; (d) Noise density $=5 \%$; (e) Noise density $=10 \%$; (f) Noise density $=20 \%$.
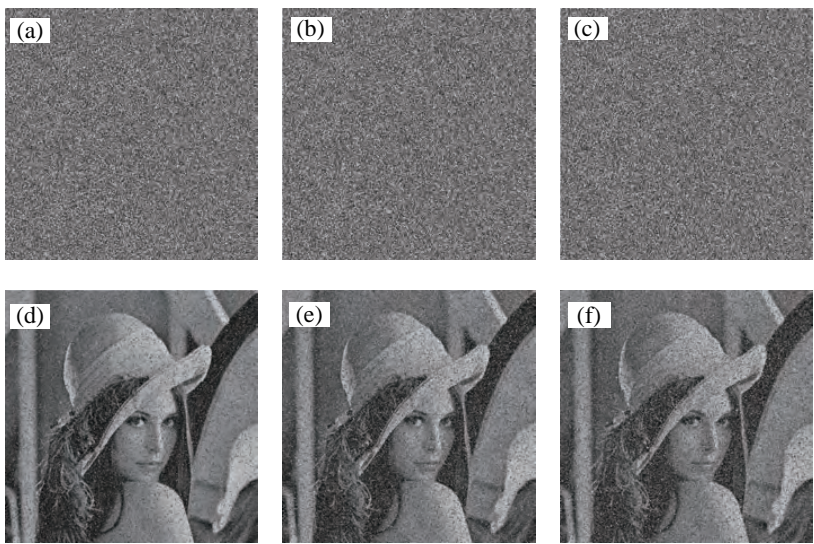

Fig. 15 Cipher- and decrypted images under Gaussian noises with different levels. (a) Mean $=0$, Variance $=0.0001$; (b) $\quad$ Mean $=0, \quad$ Variance $=0.0003 ; \quad$ (c) $\quad M e a n=0$, Variance $=0.0005 ; \quad(\mathrm{d}) \quad$ Mean $=0, \quad$ Variance $=0.0001 ; \quad(\mathrm{e})$ Mean $=0$, Variance $=0.0003$; (f) Mean $=0$, Variance $=0.0005$.
Table 8 demonstrates that the proposed parallel image encryption algorithm can achieve a fast encryption speed and also a secure system performance.

Table 7 Execution time of the proposed system (unit:ms).

\begin{tabular}{lc}
\hline Process & Time \\
\hline The quantification process & 0.5198 \\
The construction of encryption matrix & 0.3825 \\
The pixel permutation and diffusion process & 1.8610 \\
Others & 0.6732 \\
Total & 3.4365 \\
\hline
\end{tabular}

Total Encryption Time (3.4365 ms)

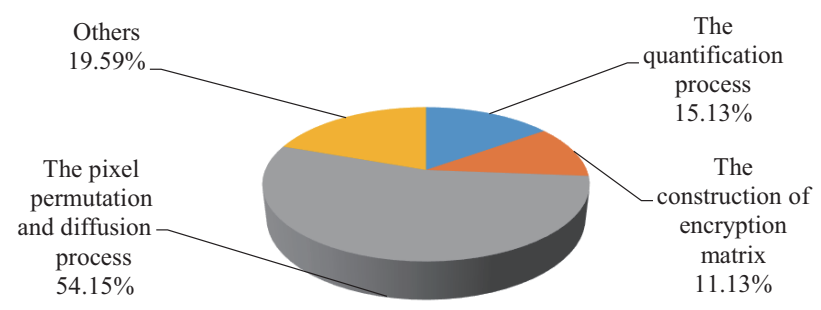

Fig. 16 Average encryption percentage time of each component of the proposed algorithm for Lena of size $512 \times$ 512 .

Table 8 Performance comparisons with other research works.

\begin{tabular}{|c|c|c|c|c|c|}
\hline Algorithms & Entropy & Key space & NPCR & UACI & $\begin{array}{l}\text { Time } \\
(m s)\end{array}$ \\
\hline Ref. 44 & 7.9971 & $10^{96}$ & $99.59 \%$ & $33.25 \%$ & 175.00 \\
\hline Ref. 31 & 7.9973 & $2^{128}$ & $99.61 \%$ & $33.36 \%$ & 119.80 \\
\hline Ref. 47 & 7.9993 & -- & $81.20 \%$ & $27.39 \%$ & 34.78 \\
\hline Ref. 1 & 7.9960 & $7.2 \times 2^{134}$ & $99.57 \%$ & $35.08 \%$ & 9.77 \\
\hline Ref. 8 & 7.8190 & $2^{448}$ & $99.63 \%$ & $31.15 \%$ & 3.14 \\
\hline Ref. 10 & 7.9993 & {$\left[\left(\mathrm{~N}^{2}\right) !\right]^{\mathrm{nm}} L^{\mathrm{nm}}$} & $99.60 \%$ & $28.60 \%$ & 7.00 \\
\hline Ref. 43 & 7.9992 & -- & $99.62 \%$ & $33.46 \%$ & 65 \\
\hline Ref. 38 & 7.9914 & $2^{561}$ & $99.61 \%$ & $33.47 \%$ & 438 \\
\hline Ref. $\overline{42}$ & -- & $10^{84}$ & $99.61 \%$ & $33.40 \%$ & 113.9 \\
\hline This work & 7.9994 & $10^{150} \times 2^{256}$ & $99.62 \%$ & $33.48 \%$ & 3.44 \\
\hline
\end{tabular}

\section{Conclusions}

In this paper, a robust parallel image encryption algorithm is proposed. It uses plain-image and external keys to design a novel decimal quantization scheme and construct an encryption matrix. Four 
chaotic sequences are constructed by the combination of the PWLCM and FDHCM. The chaotic sequences and encryption matrix are used to control the permutation and diffusion processes. The proposed algorithm is implemented based on the GPU device using the OpenCL technique. It achieves a fast computing speed. The security performance is evaluated by using key space analysis, statistical analysis, sensitivity analysis, and robustness analysis. Experimental results show that the proposed cryptosystem has a large key space and is resistant to entropy, differential and chosen/known plain-image attacks.

\section{Acknowledgements}

This research is supported by the National Natural Science Foundation of China under Grants 61661008 and 61603104, the Guangxi Natural Science Foundation under Grants 2017GXNSFAA198180, 2015GXNSFBA139256 and 2016GXNSFCA380017, the funding of Overseas 100 Talents Program of Guangxi Higher Education, the Research Project of Guangxi University of China under Grant KY2016YB059, Guangxi Key Lab of Multi-source Information Mining \& Security under Grant MIMS15-07, the Doctoral Research Foundation of Guangxi Normal University, the Innovation Project of Guangxi Graduate Education under Grant YCSZ2017055, the Scientific Research Funds for the Returned Overseas Chinese Scholars from State Education Ministry, the Funds for Young Key Program of Education Department from Fujian Province, China (Grant No. JZ160425), Program of Education Department of Fujian Province, China (Grant No. I201501005).

\section{References}

1. Abanda, Y., Tiedeu, A.: Image encryption by chaos mixing. IET Image Processing 10, 742-750 (2016)

2. Arroyo, D., Li, C., Li, S., Alvarez, G., Halang, W.A.: Cryptanalysis of an image encryption scheme based on a new total shuffling algorithm. Chaos Solitons \& Fractals 41(5), 2613-2616 (2009)

3. Chai, X., Chen, Y., Broyde, L.: A novel chaotic image encryption scheme using dna sequence operations. Optics and Lasers in Engineering 73, 53-61 (2015)

4. Chai, X.L., Gan, Z.H., Yuan, K., Lu, Y., Chen, Y.R.: An image encryption scheme based on three-dimensional brownian motion and chaotic system. Chinese Physics B 26, 020,504 (2017)

5. Chen, J., Zhu, Z., Fu, C., Zhang, L., Yu, H.: Analysis and improvement of a double-image encryption scheme using pixel scrambling technique in gyrator domains. Optics and Lasers in Engineering 66, 1-9 (2015)
6. Chen, L., Ma, B., Zhao, X., Wang, S.: Differential cryptanalysis of a novel image encryption algorithm based on chaos and line map. Nonlinear Dynamics 87(3), 1797-1807 (2017)

7. Chen, L., Wang, S.: Differential cryptanalysis of a medical image cryptosystem with multiple rounds. Computers in Biology and Medicine 65, 69-75 (2015)

8. Choi, J., Seok, S., Seo, H., Kim, H.: A fast arx modelbased image encryption scheme. Multimedia Tools and Applications 2, 14,685-14,706 (2016)

9. Dong, C.: Color image encryption using one-time keys and coupled chaotic systems. Signal Processing: Image Communication 29, 628-640 (2014)

10. Elgendy, F., Sarhan, A.M., Eltobely, T.E., El-Zoghdy, S.F., El-sayed, H.S., Faragallah, O.S.: Chaos-based model for encryption and decryption of digital images. Multimedia Tools and Applications 75, 11,529-11,553 (2016)

11. Fu, C., Meng, W.h., Zhan, Y.f., Zhu, Z.l., Lau, F.C.M., Tse, C.K., Ma, H.f.: An efficient and secure medical image protection scheme based on chaotic maps. Computers in biology and medicine 43, 1000-10 (2013)

12. Gu, G., Ling, J.: Optik a fast image encryption method by using chaotic $3 \mathrm{~d}$ cat maps. Optik - International Journal for Light and Electron Optics 125, 4700-4705 (2014)

13. Hua, Z., Zhou, Y.: Image encryption using 2d logisticadjusted-sine map. Information Sciences 339, 237-253 (2016)

14. Hua, Z., Zhou, Y.: Design of image cipher using blockbased scrambling and image filtering. Information Sciences 396, 97-113 (2017)

15. Huang, R., Rhee, K., Uchida, S.: A parallel image encryption method based on compressive sensing. Multimedia Tools and Applications 72, 71-93 (2014)

16. Huang, X., Ye, G.: An image encryption algorithm based on hyper-chaos and dna sequence. Multimedia Tools and Applications 72, 57-70 (2014)

17. Kadir, A., Hamdulla, A., Guo, W.Q.: Color image encryption using skew tent map and hyper chaotic system of 6th-order cnn. Optik 125, 1671-1675 (2014)

18. Kamel Mohamed, F.: A parallel block-based encryption schema for digital images using reversible cellular automata. Engineering Science and Technology, an International Journal 17, 85-94 (2014)

19. Lee, J., Yi, F., Saifullah, R., Moon, I.: Graphics processing unit caccelerated double random phase encoding for fast image encryption. Optical Engineering 53, 112,308 (2014)

20. Li, C., Li, S., Chen, G., Halang, W.a.: Cryptanalysis of an image encryption scheme based on a compound chaotic sequence. Image and Vision Computing 27, 1035-1039 (2009)

21. Li, C., Liu, Y., Xie, T., Chen, M.Z.Q.: Breaking a novel image encryption scheme based on improved hyperchaotic sequences. Nonlinear Dynamics $\mathbf{7 3}(3)$, 2083-2089 (2013)

22. Li, C., Liu, Y., Zhang, L.Y., Wong, K.W.: Cryptanalyzing a class of image encryption schemes based on Chinese Remainder Theorem. Signal Processing: Image Communication 29(8), 914-920 (2014)

23. Li, C., Lo, K.T.: Optimal quantitative cryptanalysis of permutation-only multimedia ciphers against plaintext attacks. Signal Processing 91, 949-954 (2011)

24. Li, C., Xie, T., Liu, Q., Cheng, G.: Cryptanalyzing image encryption using chaotic logistic map. Nonlinear Dynamics 78(2), 1545-1551 (2014) 
25. Li, Y., Wang, C., Chen, H.: A hyper-chaos-based image encryption algorithm using pixel-level permutation and bit-level permutation. Optics and Lasers in Engineering 90, 238-246 (2017)

26. Liu, Y., Fan, H., Xie, E.Y., Cheng, G., Li, C.: Deciphering an image cipher based on mixed transformed logistic maps. International Journal of Bifurcation and Chaos 25(13), art. no. 1550,188 (2015)

27. Luo, Y., Cao, L., Qiu, S., Lin, H., Harkin, J., Liu, J.: A chaotic map-control-based and the plain image-related cryptosystem. Nonlinear Dynamics 83, 2293-2310 (2016)

28. Luo, Y., Du, M., Liu, J.: A symmetrical image encryption scheme in wavelet and time domain. Communications in Nonlinear Science and Numerical Simulation 20, 447-460 (2014)

29. Matthews, R.: on the derivation of a chaotic encryption algorithm. Cryptologia 13(1), 29-42 (1989)

30. Mirzaei, O., Yaghoobi, M., Irani, H.: A new image encryption method: Parallel sub-image encryption with hyper chaos. Nonlinear Dynamics 67, 557-566 (2012)

31. Murillo-Escobar, M., Cruz-Hernández, C., AbundizPérez, F., López-Gutiérrez, R., Del Campo, O.A.: A rgb image encryption algorithm based on total plain image characteristics and chaos. Signal Processing 109, 119131 (2015)

32. Qiu, H., Memmi, G.: Fast selective encryption method for bitmaps based on gpu acceleration. 2014 IEEE International Symposium on Multimedia pp. 155-158 (2014)

33. Saikumar, N.: An encryption approach for security enhancement in images using key based partitioning technique. In: 2016 International Conference on Circuit, Power and Computing Technologies [ICCPCT], pp. 1-3 (2016)

34. Singh, H., Yadav, A.K., Vashisth, S., Singh, K.: Double phase-image encryption using gyrator transforms, and structured phase mask in the frequency plane. Optics and Lasers in Engineering 67, 145-156 (2015)

35. Wang, Q., Yu, S., Li, C., Lü, J., Fang, X., Guyeux, C., Bahi, J.M.: Theoretical design and FPGA-based implementation of higher-dimensional digital chaotic systems. IEEE Transactions on Circuits and Systems I-Regular Papers 63(3), 401-412 (2016)

36. Wang, X., Yang, L., Liu, R., Kadir, A.: A chaotic image encryption algorithm based on perceptron model. Nonlinear Dynamics 62(3), 615-621 (2010)

37. Wei, X., Guo, L., Zhang, Q., Zhang, J., Lian, S.: A novel color image encryption algorithm based on dna sequence operation and hyper-chaotic system. Journal of Systems and Software 85, 290-299 (2012)

38. Wu, X., Wang, D., Kurths, J., Kan, H.: A novel lossless color image encryption scheme using 2D DWT and 6D hyperchaotic system. Information Sciences 349-350, 137$153(2016)$

39. Xie, E.Y., Li, C., Yu, S., Lü, J.: On the cryptanalysis of Fridrich's chaotic image encryption scheme. Signal Processing 132, 150-154 (2017)

40. Xie, K., Wu, P., Yang, S.: Gpu and cpu cooperation parallel visualisation for large seismic data. Electronics Letters 46, 1196 (2010)

41. Yaghouti Niyat, A., Moattar, M.H., Niazi Torshiz, M.: Color image encryption based on hybrid hyper-chaotic system and cellular automata. Optics and Lasers in Engineering 90, 225-237 (2017)

42. Ye, G.D., Huang, X.L., Zhang, L.Y., Wang, Z.X.: A selfcited pixel summation based image encryption algorithm. Chinese Physics B 26, 010,501 (2017)
43. Zhang, W., Yu, H., Zhao, Y., Zhu, Z.: Image encryption based on three-dimensional bit matrix permutation. Signal Processing 118, 36-50 (2016)

44. Zhang, Y.Q., Wang, X.Y.: A chaotic image encryption algorithm based on perceptron model. Nonlinear Dynamics 77, 687-698 (2014)

45. Zheng, Y., Jin, J.: A novel image encryption scheme based on henon map and compound spatiotemporal chaos. Multimedia Tools and Applications 74, 7803-7820 (2014)

46. Zhu, C.: A novel image encryption scheme based on improved hyperchaotic sequences. Optics Communications 285, 29-37 (2012)

47. Zhu, Z., Zhang, W., Wong, K., Yu, H.: A chaosbased symmetric image encryption scheme using a bitlevel permutation. Information Sciences 181, 1171-1186 (2011) 